\title{
SY41-4 Symposium
}

\section{The First New Botanical Drug Approved in Taiwan--PG2 Injection}

\section{Chien-Hsin D. Cheng}

\section{PhytoHealth Corporation, Taiwan}

PG2, different from single-ingredient chemically synthetic drugs, is a novel lyophilized injection containing complicated mixture of plant-derived ingredients. PG2's raw materials, Astragalus membranaceus, were cultivated in the specific region of Inner Mongolia, China. Based on the GAP (Good Agricultural Practice) protocols, Astragalus were grown via breeding, nurturing seedlings, field growing, harvesting to monitor soil, water, fertilizer in order to control their qualities and batch-to-batch uniformity. After harvesting, Astragalus underwent step-by-step SOP such as screening, washing, drying, slicing before shipping to Taiwan. Every batch of Astragalus raw material must go through DNA appraisal to confirm it was Astragalus membranaceus by Industrial Technology Research Institute (ITRI), Hsinchu, Taiwan. Astragalus were subsequently manufactured through a series of proprietary processes: extraction, condensation, filtration, purification and spray drying to obtain the highly-purified polysaccharides as the PG2 active pharmaceutical ingredients (API). The entire process from raw material to finished product comprised of 68 steps and 141 inspection items to ensure product quality and uniformity.

Following TFDA's "Guidance for Botanical Drug Clinical Trials" and "Guidance for Botanical Drug New Drug Application", PG2 underwent complete animal toxicology studies and Phases I, II and III human clinical trials before submitting for NDA with the TFDA. This presentation will demonstrate the positive Phase III clinical study outcomes together with the results from an even larger-scale Phase IV post-marketing clinical trials. PG2 was finally approved by the TFDA for treating cancer-related fatigue (CRF), a disease that is seriously associated with chemotherapy and immunotherapy among cancer patients. PG2 has been marketed to more than 30 major medical centers, hospitals, clinics and has treated more than 5,000 late-stage cancer patients in Taiwan. 